\title{
Cushing disease: use of perioperative serum cortisol measurements in early determination of success following pituitary surgery
}

\author{
Armand Krikorian, M.D., ${ }^{1}$ Dima AbdelMannan, M.D., ${ }^{1}$ Warren R Selman, M.D., ${ }^{2}$ \\ AND BAHA M ARAFAH, M.D. ${ }^{1}$ \\ ${ }^{1}$ Division of Endocrinology and ${ }^{2}$ Neurological Institute, University Hospitals/Case Medical Center, \\ Case Western Reserve University, Cleveland, Ohio
}

\begin{abstract}
$\checkmark$ Despite many recent advances, management of cases of Cushing disease continues to be challenging. After complete resection of ACTH-secreting adenomas, patients develop transient ACTH deficiency requiring glucocorticoid replacement for several months. The current recommendation by many centers, including ours, for patients with ACTHsecreting adenomas is to withhold glucocorticoid therapy during and immediately after adenomectomy until there is clinical or biochemical evidence of ACTH deficiency. A serum cortisol level of less than $2 \mu \mathrm{g} / \mathrm{dl}$ within the first 48 hours after adenomectomy is a reliable biochemical marker of ACTH deficiency and is associated with clinical remission of Cushing disease. Higher serum cortisol levels in the immediate postoperative period should be interpreted with caution. The decision to immediately reexplore the sella turcica should be individualized, taking into account the findings at surgery, the histopathological findings, and the changes in serum cortisol levels as well as the patient's wishes and concerns. Optimal diagnosis and therapy for patients with Cushing disease require thorough and close coordination and involvement of all members of the management team. (DOI: 10.3171/FOC-07/09/E6)
\end{abstract}

\section{Key Words • adenoma • cortisol • Cushing disease • Cushing syndrome • pituitary surgery}

$\mathrm{C}$ USHING syndrome refers to a state of hypercortisolism (endogenous or exogenous) regardless of its etiology. Cushing disease defines a state of hypercortisolism caused by an ACTH-secreting pituitary adenoma. These tumors represent approximately 10 to $12 \%$ of all pituitary adenomas and are seen predominantly in women, with a female to male ratio of $8: 1$ and a peak incidence in the third to fourth decades of life. The ACTH-secreting pituitary adenomas represent the most common cause of endogenous hypercortisolism; accounting for approximately 65 to $70 \%$ of all cases of Cushing syndrome. ${ }^{3}$ While most such tumors are generally benign, some are more invasive than other pituitary adenomas. Although most patients with ACTH-secreting adenomas present with small tumors that may not even be evident on magnetic resonance imaging, some have invasive macroadenomas. In addition to signs and symptoms of hypercortisolism, the clinical manifestations in the latter group of patients in- clude signs of mechanical effects of the macroadenoma on surrounding structures. Such effects include headaches, visual symptoms, and impairment of normal pituitary function. ${ }^{3}$

Even though the clinical, biochemical, and imaging characteristics of cases of Cushing disease have been well appreciated for decades, the diagnosis and management of this disease are often challenging. ${ }^{11}$ Patients with Cushing disease exhibit increased rates of mortality and morbidity secondary to chronic hypercortisolism..$^{12}$ Deleterious effects of hypercortisolism include hypertension, obesity, osteoporosis, fractures, and impaired immune function and wound healing as well as glucose intolerance and diabetes. The high morbidity and mortality rates associated with this disease make it imperative to optimize its management.

The care of patients with Cushing disease requires attention to details and integration of clinical, biochemical, and imaging data. Although the therapeutic options for managing the disease include several approaches, the consensus is that transsphenoidal adenomectomy is the treatment of choice. In the hands of an experienced neurosurgeon, this approach offers remission rates between 70 and 


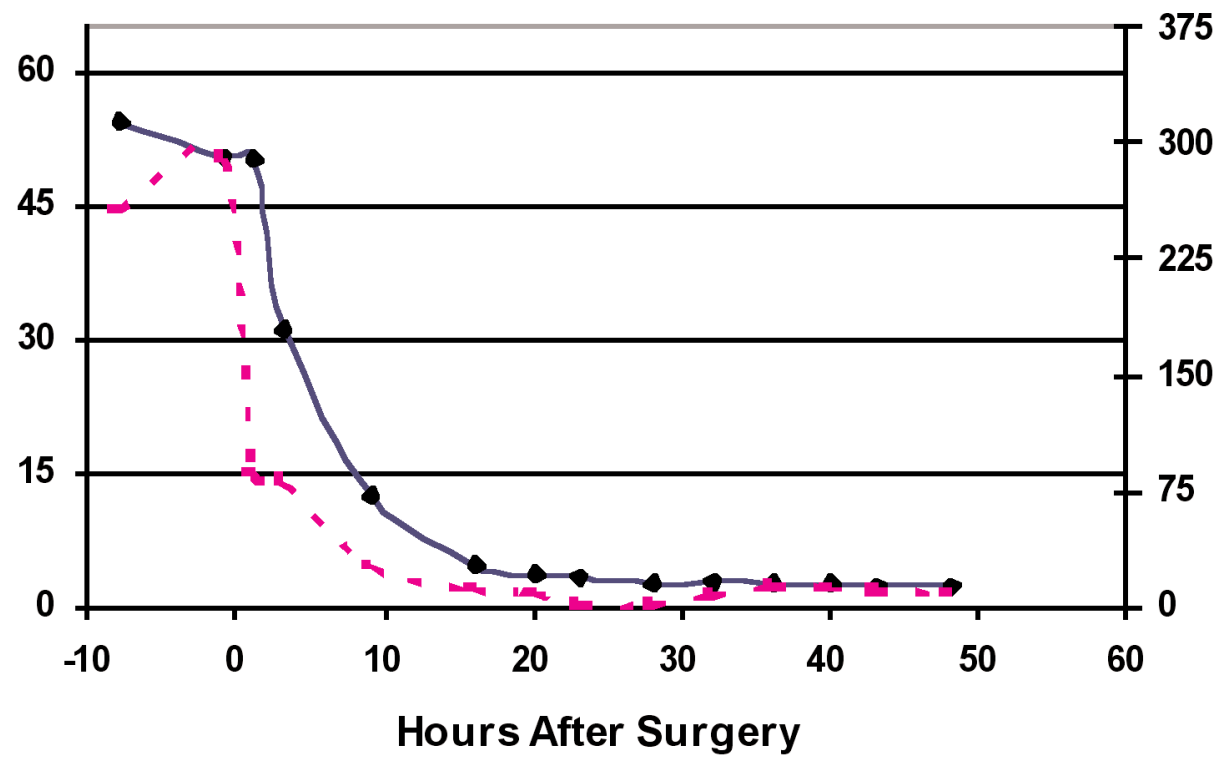

FIG. 1. Serial measurements of serum cortisol (solid line) and plasma ACTH levels (interrupted line) obtained before and immediately after adenomectomy (Time 0) in a patient with histologically documented ACTH-secreting pituitary adenoma. The plasma ACTH level decreased to less than $10 \mathrm{ng} / \mathrm{L}$ approximately 16 to 18 hours after surgery while serum cortisol levels dropped at a slower rate, reaching values of 2 to $4+\mu \mathrm{g} / \mathrm{dl}$ nearly 20 hours following adenomectomy. Ascending numbers at left represent $\mu \mathrm{g} / \mathrm{dl}$ of cortisol; those at right ng/L of ACTH (this applies to Figs. 2-4 as well).

$85 \%$ with mortality and morbidity rates less than $2 \% .25$ Other therapeutic options for the management of patients with ACTH-secreting pituitary adenoma include treatment with adrenostatic medications, pituitary irradiation, and bilateral adrenalectomy. These last options are often used in cases in which pituitary adenomectomy has failed. ${ }^{24}$

The perioperative management of patients with ACTHsecreting adenomas is not uniform. While some centers have abandoned the routine administration of glucocorticoids during the first few hours after surgery, many continue to use glucocorticoids during and after surgery. Similarly, while some centers define early remission as a perioperative serum level of less than $2 \mu \mathrm{g} / \mathrm{dl},{ }^{16}$ others use a serum level of less than $5 \mu \mathrm{g} / \mathrm{dl}$ as an indication of early success. ${ }^{29}$ Some authors ${ }^{25}$ have also used the perioperative serum cortisol levels as a guide for early intervention and perhaps reexploration of the sella turcica.

In this report, we review the changes in cortisol secretion that occur after pituitary adenomectomy and discuss the rationale and timing of withholding glucocorticoid therapy during and after adenomectomy. We also discuss the limitations of serum cortisol measurements and the impact on case management.

\section{Perioperative Glucocorticoid Administration}

\section{Patients With Non-ACTH Secreting Adenomas}

The administration of glucocorticoids to all patients undergoing pituitary adenomectomy was routinely practiced in the 1970s and 1980s, regardless of adenoma type. The rationale for this practice was the unsubstantiated concern for the potential damage to the normal gland. Several studies conducted in patients with pituitary adenomas during and after selective adenomectomy demonstrated that the HPA function is highly activated. ${ }^{18}$ This was evidenced by appropriately elevated plasma ACTH and cortisol levels. ${ }^{1-3}$ Over the years, many centers have abandoned the routine use of glucocorticoids during pituitary adenomectomy.

Patients with preoperative ACTH deficiency will obviously need glucocorticoid therapy, at least during and perhaps immediately after selective adenomectomy. ${ }^{16}$ Several studies have documented the immediate recovery of HPA function after selective removal of larger adenomas with the subsequent recovery of other pituitary function thereafter. ${ }^{1,2}$ Many such patients who had documented hypopituitarism preoperatively recover at least partial HPA function immediately after adenomectomy and are discharged without replacement therapy. ${ }^{1,2,16,36}$

\section{Patients With ACTH-Secreting Adenomas}

The HPA function in patients with ACTH-secreting adenomas is distinctly different from that of others harboring different types of tumors. In patients with ACTH-secreting adenomas, autonomous secretion of ACTH by the tumor stimulates excessive and persistent increases in cortisol secretion. The latter persistent state of hypercortisolism causes suppression of normal pituitary corticotrophs. Thus, because the normal corticotrophs have been suppressed, patients exhibit signs and symptoms of ACTH deficiency shortly after an ACTH-secreting adenoma is completely resected. In these patients ACTH deficiency is transient, often lasting 6 to 18 months. ${ }^{16}$ In fact, the development of ACTH deficiency after removal of a pituitary corticotroph adenoma is a favorable sign that often indicates the high likelihood for remission of Cushing disease.

Over 25 years ago, we reasoned that instead of treating patients with Cushing disease with glucocorticoids, one could monitor the development of ACTH deficiency in the immediate postoperative period. ${ }^{4}$ At that time, serum cortisol measurements were not as readily available as they are 


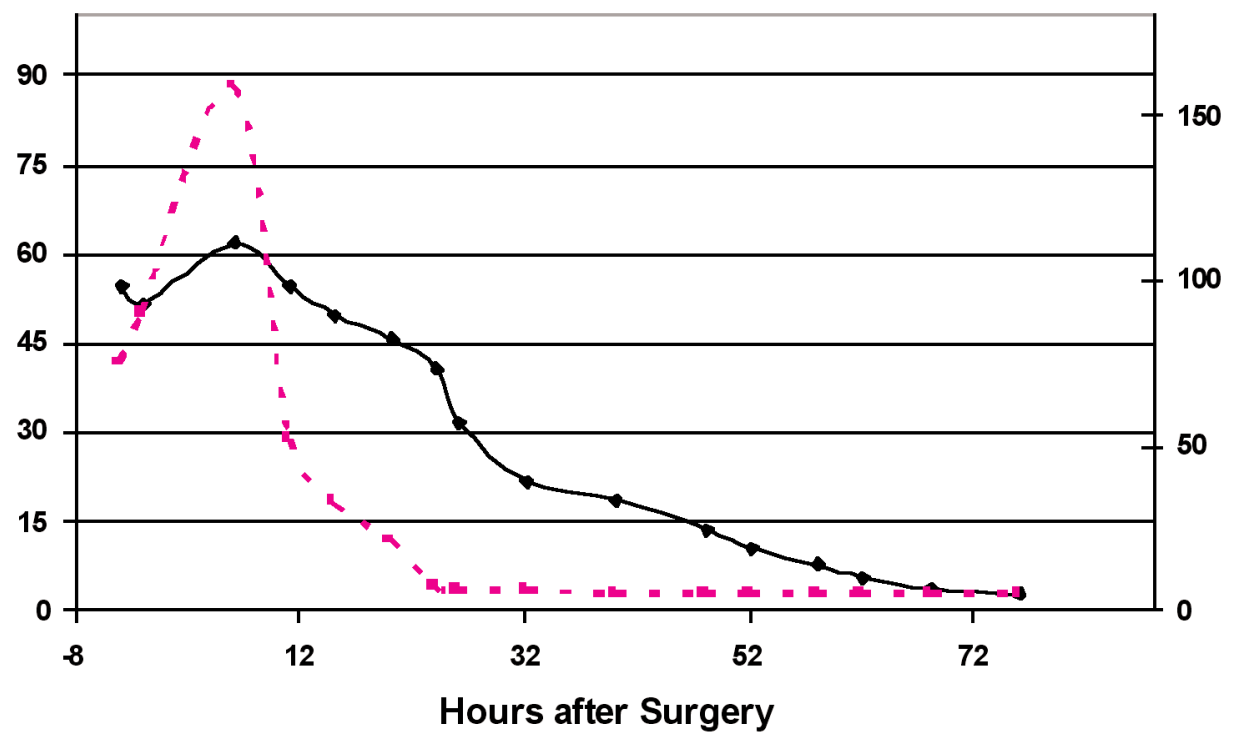

FIG. 2. Serial measurements of serum cortisol (solid line) and plasma ACTH levels (interrupted line) obtained before and immediately after adenomectomy (Time 0) in a postmenopausal woman with histologically documented ACTHsecreting pituitary adenoma. The patient was receiving estrogen therapy at the time of adenomectomy. The drop in the plasma ACTH levels was similar to that seen in Fig. 1. In contrast, serum cortisol levels decreased at a much slower rate, reaching values of 2 to $4 \mu \mathrm{g} / \mathrm{dl}$ nearly 72 hours after adenomectomy.

now. The results were usually available 2 or 3 days after samples were drawn. For that reason, such patients were monitored very carefully in the intensive care unit. They were given glucocorticoids whenever they exhibited any symptoms suggestive of adrenal insufficiency-without the wait for the serum cortisol concentrations. It is quite important to note that most patients whose serum cortisol levels were less than $5 \mu \mathrm{g} / \mathrm{dl}$ had minimal to moderate symptoms and were hemodynamically stable during the first 48 hours after adenomectomy. We noted that serum cortisol levels drop to less than $3 \mu \mathrm{g} / \mathrm{dl}$ approximately 30 to 36 hours after complete adenomectomy. A typical graph of the changes in serum cortisol and plasma ACTH levels in the immediate postoperative period is presented in Fig. 1. The graph shows that although plasma ACTH levels decline rapidly after adenomectomy, they are still measurable during the first 12 hours. This observation is supported by data published by Graham and colleagues,${ }^{14}$ who measured plasma ACTH levels during the first 60 minutes after adenomectomy. The authors noted that 1 hour after adenomectomy plasma ACTH levels decreased by $54 \%$ in patients who were subsequently considered cured. The authors also noted that although patients who were not cured had a lower percentage drop in their plasma ACTH levels during the first postoperative hour, it was difficult to predict cure on the basis of ACTH measurements at that time. Thus, based on these data and recognizing the half-life of cortisol, it would be reasonable to state that serum cortisol levels remain normal or high during and immediately after surgical adenomectomy.

These data along with ours argue strongly against the necessity of administering glucocorticoids to patients with ACTH-secreting adenomas during or immediately after adenomectomy. Several centers have reported successfully withholding routine corticosteroid administration to pa- tients with Cushing disease during and immediately after adenomectomy. ${ }^{10,16,25,29}$ Indeed, in a comparison of serum cortisol levels in patients achieving remission from Cushing disease and those who did not, a drop in serum cortisol levels started to occur 6 hours postoperatively and did not become statistically different between the two groups until 12 hours after adenomectomy. ${ }^{29}$ In another study of 27 patients with Cushing disease, ${ }^{30}$ all patients were not given routine perioperative glucocorticoids and those achieving remission did not develop clinical symptoms of adrenal insufficiency until the first postoperative day.

Nevertheless, review of current literature shows that routine perioperative administration of corticosteroids in patients undergoing pituitary adenomectomy for Cushing disease is still a common and widely reported practice. ${ }^{19-23}$ Thus, despite the state of endogenous hypercortisolism associated with Cushing disease, many such patients are still unnecessarily given exogenous glucocorticoids during pituitary surgery.

In summary, close monitoring of serum cortisol postoperatively allows the detection of those patients who will need glucocorticoid replacement. We advocate measurements of serum cortisol every 6 hours starting immediately after adenomectomy and continuing for 3 days postoperatively. We also advocate the administration of hydrocortisone replacement when signs and symptoms of adrenal insufficiency develop and/or when the serum cortisol is less than $2 \mu \mathrm{g} / \mathrm{dl}$.

\section{Perioperative Assessment of Surgical Outcome}

Several methods have been used to define remission after removal of ACTH-secreting adenomas. These methods include serum cortisol measurements, 24-hour urinary 


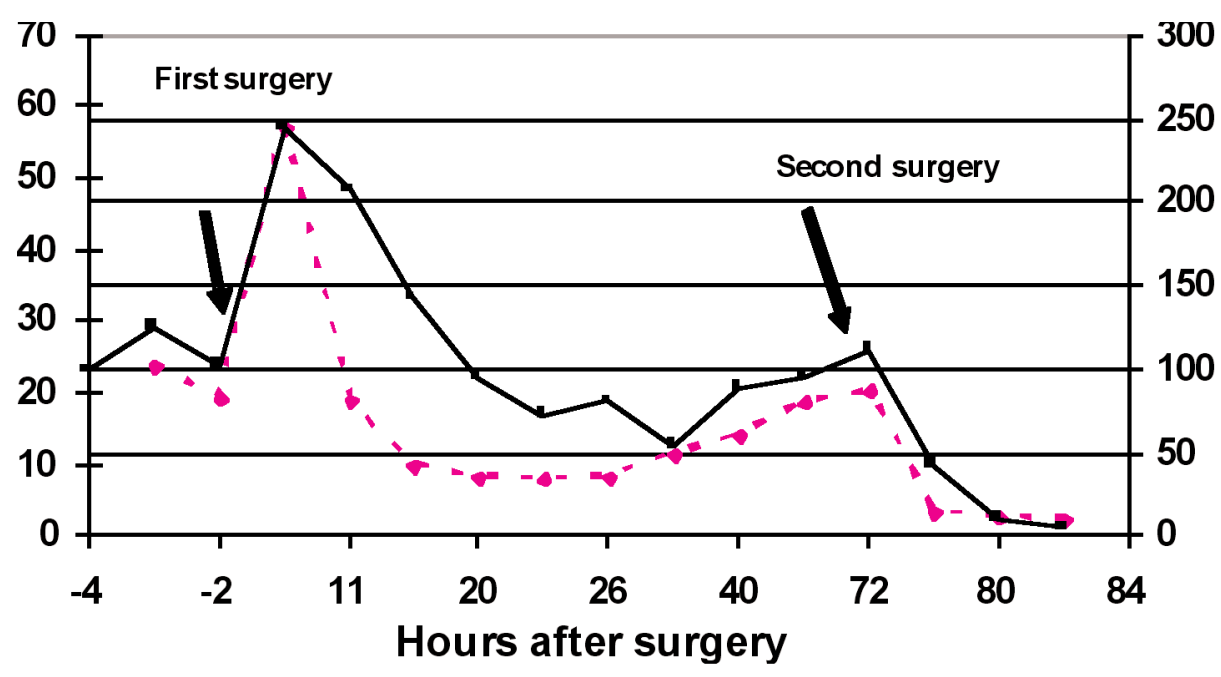

FIG. 3. Serial measurements of serum cortisol (solid line) and plasma ACTH levels (interrupted line) obtained before and immediately after adenomectomy (Time 0 ) in a young man with histologically documented ACTH-secreting pituitary adenoma. After the first surgery, the serum cortisol and plasma ACTH values were decreased but not to the expectedly low levels noted after complete adenomectomy. After confirmation of the presence of corticotroph adenoma on the first surgical procedure, it was felt that the adenomectomy was incomplete and therefore re-exploration of the sella was performed soon thereafter. The changes in serum cortisol and plasma ACTH after the second surgical procedure were similar to those observed after complete adenomectomy as shown in Fig. 1.

free cortisol determination, the 1-mg dexamethasone suppression test, and dexamethasone suppression combined with corticotropin-releasing hormone administration., ${ }^{5,826}$ Of these options, the simplest to perform is measuring serum cortisol levels frequently and directly after surgery. As stated earlier, normal pituitary corticotrophs are suppressed in patients with ACTH-secreting adenomas. This fact becomes evident shortly after complete adenomectomy, when plasma ACTH and serum cortisol levels decline quickly. In many centers, including our own, patients are monitored for the development of adrenal insufficiency in the immediate postoperative period. In such instances, the development of adrenal insufficiency is a good prognostic sign indicating possible complete adenomectomy and a high likelihood of remission; the suppression of the HPA axis often lasts several months, and in these cases glucocorticoid replacement is required for 9 to 12 months.

The sparing use of perioperative glucocorticoids permits early assessment of remission after adenomectomy by direct measurements of serum cortisol levels. A serum cortisol level of less than $2 \mu \mathrm{g} / \mathrm{dl}$ in the early postoperative phase has been reported to be an excellent prognostic indicator associated with long-term remission. ${ }^{33,34}$ The nadir serum cortisol levels seem to occur by 48 hours postoperatively in most patients. ${ }^{16}$ As a corollary, the absence of hypocortisolism in the postoperative period is associated with a recurrence rate of up to $67 \% .{ }^{11}$ Some authors have advocated the use of a cut-off value of $5 \mu \mathrm{g} / \mathrm{dl}$ to define remission in the immediate postoperative period. ${ }^{10,15}$ The authors of one study reported that disease recurred in one (4\%) of 25 patients with a postoperative plasma cortisol level of less than $2 \mu \mathrm{g} / \mathrm{dl}{ }^{20}$ In a retrospective study, ${ }^{35}$ disease recurred in $11.5 \%$ of patients who had undetectable cortisol levels postoperatively. One limitation of this study was the different detection ranges of the cortisol assays used over the years. Nevertheless, it can be inferred that the use of a higher cutoff value may lead to decreased specificity of the serum cortisol for predicting recurrence. It is very important to emphasize the fact that a drop in serum cortisol to less than $2 \mu \mathrm{g} / \mathrm{dl}$ in the immediate postoperative period is clearly associated with remission but is still associated with a small $(10 \%)$ probability of recurrence in the future.

As stated earlier, other approaches to assessing the outcome of resection in the perioperative period have been reported. ${ }^{5,8,26}$ Data on urinary free cortisol measurements show a statistically significant difference between remission and treatment failure groups only after 6 weeks after surgery. ${ }^{7}$ The combination of the dexamethasone suppression test and administration of corticotropin-releasing hormone is limited by low specificity. ${ }^{5} \mathrm{~A}$ report by Chen and colleagues $^{8}$ evaluated the utility of overnight suppression of HPA function with dexamethasone $(1 \mathrm{mg})$ in the assessment of surgical outcome in patients with Cushing disease. In the latter study, Chen and colleagues ${ }^{8}$ determined serum cortisol levels on the third postoperative day, after having administered dexamethasone $(1 \mathrm{mg})$ the night before the measurement. The authors reported a $93 \%$ 5-year remission rate in the patients who had a serum cortisol level of less than $3 \mu \mathrm{g} / \mathrm{dl}$ the morning after dexamethasone administration. Although this approach to assessment might be reasonable, it does not offer any significant advantage over the previously discussed protocol that avoids the use of dexamethasone. It is well known that many patients with Cushing disease show partial suppression of HPA axis function after dexamethasone administration. The phenomenon might result in misleadingly lower serum cortisol, particularly in patients with incomplete tumor resection who are also sensitive or responsive to dexamethasone. 


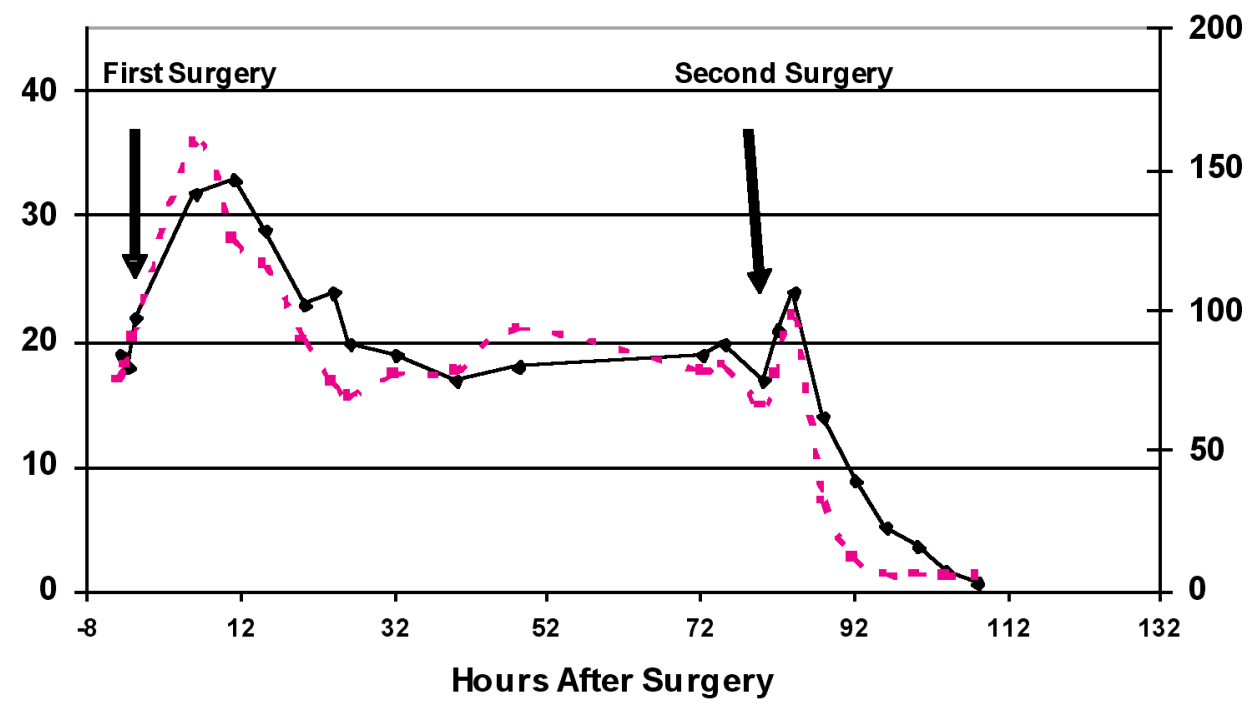

FIG. 4. Serial measurements of serum cortisol (solid line) and plasma ACTH levels (interrupted line) obtained before and immediately after adenomectomy (Time 0) in a woman with Cushing disease. At the first surgical procedure, an adenoma measuring $1.2 \mathrm{~cm}$ was removed; on subsequent testing it proved to be not an ACTH-secreting lesion. Reexploration of the sella demonstrated the presence of a 2-mm adenoma on the other side of the pituitary; this second tumor was demonstrated to be an ACTH-secreting lesion. A transient rise in the concentrations of ACTH and cortisol was noted for a few hours after the first surgical procedure, after which the levels returned to their respective baseline. In contrast, after the second surgical procedure, both levels decreased rapidly as would be expected after complete removal of an ACTHsecreting adenoma.

\section{Limitations to the Use of Serum Cortisol Levels}

Several factors limit the interpretation of serum cortisol levels in the immediate postoperative period. These limitations should be considered in interpreting data on serum cortisol measurements. One such limitation is the impact of changes in corticosteroid-binding globulin. All commercially available assays used for the determination of serum cortisol concentrations measure the total serum cortisol levels. Because over $90 \%$ of the serum cortisol is found in the protein-bound form, abnormalities in the major binding protein (corticosteroid-binding globulin, or transcortin) have a major impact on measured total cortisol concentrations. ${ }^{17}$ High-estrogen states such as are found in pregnancy or in patients treated with estrogen (as in postmenopausal estrogen replacement therapy or oral contraception) can lead to an increase in transcortin and a subsequent rise in measured serum cortisol concentrations. ${ }^{9}$

The impact of increased transcortin becomes more apparent and relevant when serum cortisol levels are determined in patients with ACTH-secreting adenomas shortly after adenomectomy. One such example is shown in Fig. 2: The patient had classical features of Cushing disease and was being treated with postmenopausal estrogen replacement therapy at the time of surgical adenomectomy. The graph shows that the drop in plasma ACTH in that patient is similar to that illustrated in Fig. 1 for a typical patient after adenomectomy. The decline in serum cortisol levels was slower, however, in the patient receiving estrogen (Fig. 2) than was seen in the typical patient (Fig. 1). Failure to acknowledge the effect of estrogens in elevating corticosteroid-binding globulin levels would have led to misinterpretation of the test results. Hepatitis constitutes another condition in which globulin levels, including corticoste- roid-binding globulin, are often elevated and hence lead to an elevated total cortisol level. ${ }^{27}$ Ketoconazole has been used in the pharmacological treatment of some patients with Cushing disease. ${ }^{31}$ In the adrenals, ketoconazole inhibits cytochrome P450 11B1, but it also inhibits cytochrome P450 11A1. It may also inhibit ACTH secretion by impairing corticotroph adenylate cyclase activation as was shown in vitro in rat anterior pituitary cells. ${ }^{32}$ Thus, both ACTH and serum cortisol measurements in a patient being treated with ketoconazole must be interpreted with caution.

\section{Approach in Patients With Postoperative Serum Cortisol Levels Over $2 \mu \mathrm{g} / \mathrm{dl}$}

Several possibilities should be considered whenever a patient's serum cortisol level does not fall to below $2 \mu \mathrm{g} / \mathrm{dl}$ after apparent complete adenomectomy. The possibility of elevated serum transcortin levels should be considered if the serum cortisol value is decreased to levels between 3 and $10 \mu \mathrm{g} / \mathrm{dl}$ at 48 hours after surgery, especially in the presence of signs and symptoms of adrenal insufficiency. Measuring the free serum cortisol level would be helpful but the results might not be available for several days. In the presence of symptoms of adrenal insufficiency, glucocorticoid replacement therapy should be initiated. In the absence of such symptoms, observation would be warranted as other possibilities should be considered. One such possibility is incomplete resection of the adenoma. An example of such a situation is illustrated in Fig. 3. In that instance, reexploration of the sella was undertaken after confirmation of the presence of an adenoma secreting ACTH.

If the serum cortisol level remains clearly elevated, other 


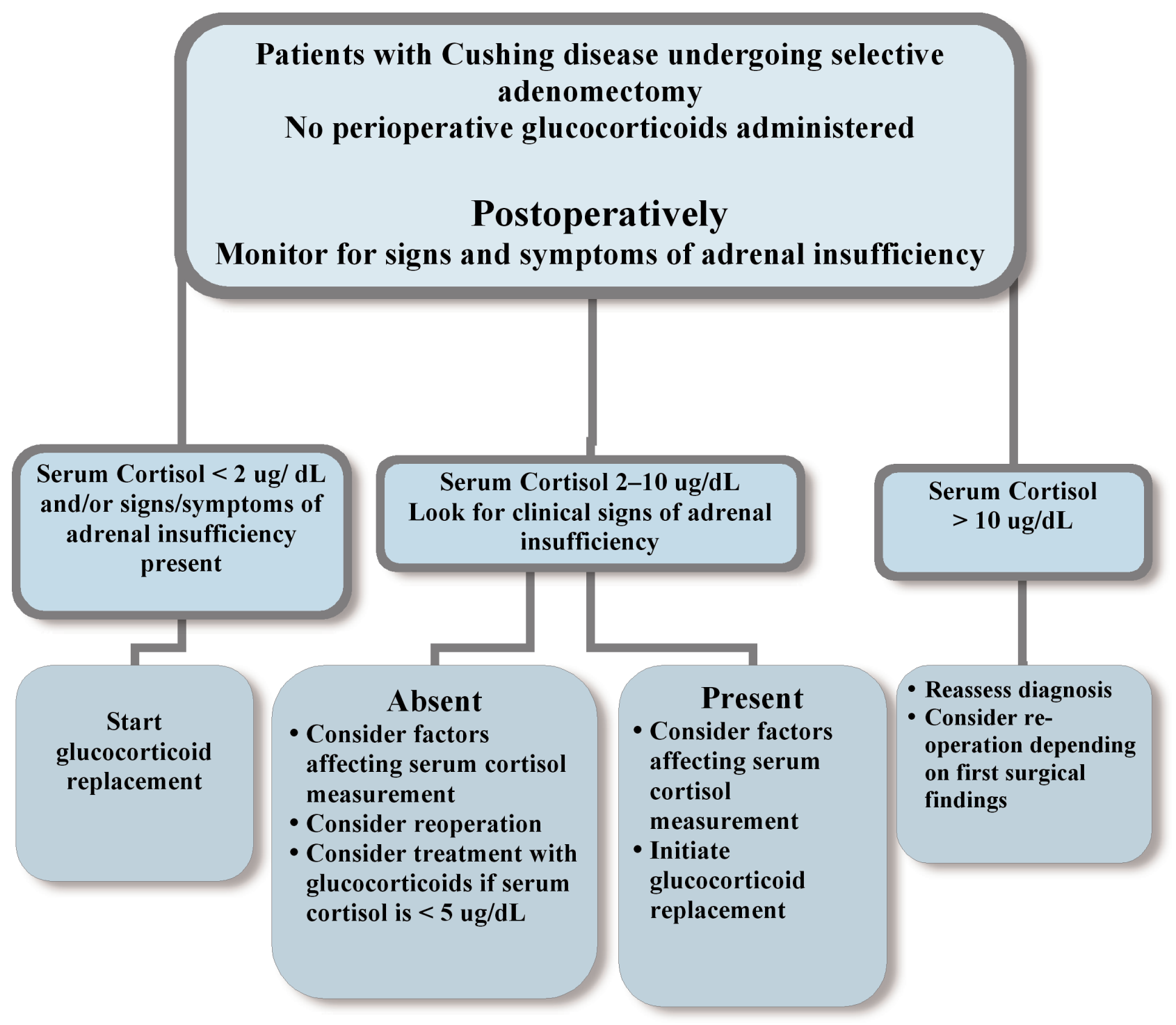

FIG. 5. Algorithm for perioperative management of patients with Cushing disease. The algorithm takes into account clinical symptoms, serum cortisol levels, and surgical findings. As emphasized in the text, additional considerations should include histopathological findings and the patient's desires and concerns.

considerations should be raised. If no adenoma was found at surgery, one should question the accuracy of the diagnosis. If, on the other hand, an adenoma was seen and resected at surgery, then one should confirm whether that adenoma was ACTH secreting or not. Finding an adenoma in the sella does not necessarily mean that it is the source of ACTH hypersecretion. This scenario is illustrated in Fig. 4. In that case, the clinical and biochemical features of Cushing disease were classic, and the magnetic resonance imaging study showed a $1.2-\mathrm{cm}$ adenoma. At surgery, the neurosurgeon removed the obvious adenoma without complications and did not thoroughly inspect the other side of the pituitary. Figure 4 illustrates the laboratory findings after surgery in that case. There was a noticeable rise in plasma ACTH and serum cortisol concentrations followed by a return to preoperative values shortly after surgery. Reexploration of the sella was quickly decided upon when the immunostaining of the resected adenoma showed that it was an incidental adenoma and not an ACTH-secreting lesion. A 2-mm adenoma was found and removed during the second surgery.

Another possibility to consider is that the diagnosis might be inaccurate. Specifically, pseudo-Cushing syndrome as well as nonpituitary causes of hypercortisolism such as ectopic ACTH secretion should be considered. In cases in which the resected adenomatous tissue stained positive for ACTH, the lack of drop in serum cortisol level to less than $2 \mu \mathrm{g} / \mathrm{dl}$ often suggests incomplete adenomectomy, provided that the previously discussed limitations and concerns have been carefully addressed. This finding 
often raises the questions of reexploration and the best timing for repeated surgery. This issue will be discussed in the following section.

\section{Early Reoperation for Cushing Disease}

Success rates, as determined by remission of Cushing disease, after initial resection of ACTH-secreting adenomas have been reported to reach $91 \% .{ }^{19}$ Mortality rates are increased in patients in whom surgery fails, whereas in patients who experience early remission the mortality rates are comparable to those in the age- and sex-matched general population. ${ }^{15}$ Thus early treatment of incompletely resected lesions appears to be a reasonable approach. In a retrospective review of 96 cases in which patients required repeated transsphenoidal surgery, with a mean interval between the two surgical procedures of 44 weeks, an initial remission was reported in $57 \%$ and a sustained remission was maintained in $43 \%$ of patients with Cushing disease. ${ }^{6}$ In another study in which the reexploration was performed 7 to 46 days after initial surgery, an improved initial remission rate of $71 \%$ was noted. ${ }^{28}$ Similar outcomes were reported when repeated surgery for Cushing disease was performed within 15 days of the first operation (67\% remission rate)..$^{25}$

Early surgical reexploration offers the added advantage of little distortion in anatomy and lack of fibrosis related to the initial procedure. These success rates after reoperation are achieved at the expense of an increased risk of hypopituitarism. ${ }^{13}$ Considering the high rate of morbidity associated with Cushing disease, repeated surgery would be a reasonable choice unless fertility is an important consideration for the patient involved. For that reason, the recommendation for reexploration should be thoroughly discussed among all members of the management team and with the patient. The extent of resection at the time of reexploration is individualized and depends on the clinical setting, the findings at the first procedure, and the patient's desires and possible interest in future fertility. This last point is particularly important because the second surgical procedure can lead to partial or complete loss of pituitary function. Despite many recent advances, restoration of fertility in patients with hypopituitarism is quite challenging and can be very expensive. In cases of incomplete adenoma resection, a hemihypophysectomy is the preferred option. An example of such a situation is illustrated in Fig. 3. In our experience, the success of hemihypophysectomy, as a second procedure, in inducing remission is approximately $65 \%$, which is comparable to other investigators' published experience. . $^{6,25,28}$

A total hypophysectomy is generally recommended and planned for patients who have already had a hemihypophysectomy without any demonstrable pituitary abnormality on the first surgical procedure, provided the risk of hypopituitarism is acceptable. Nevertheless, the success rate for total hypophysectomy after an initial hemihypophysectomy is certainly less impressive and is close to $50 \%$ at our center, although it must be acknowledged that this rate is based on only a small number of cases. It is our policy to reexamine all available data to confirm the pituitary origin of ACTH hypersecretion before recommending a total hypophysectomy. Over the past 10 years, we encountered four patients who had classical clinical and biochem- ical features of Cushing disease in whom a pituitary source was demonstrated by a 10 - to 20 -fold gradient increase in plasma ACTH levels during inferior petrosal sinus catheterization. These four patients had initially undergone a hemihypophysectomy because no obvious tumor had been noted. In two of the patients, a total hypophysectomy resulted in a remission even though no histological documentation of an adenoma was achieved. These two patients have been followed up for 6 and 8 years without evidence of recurrence. In the remaining two patients, a total hypophysectomy did not influence ACTH secretion but resulted in loss of all other pituitary function. Thus, as demonstrated in these two examples, total hypophysectomy might not benefit all patients with a presumed pituitary source of ACTH. Theoretically, a tumor secreting ACTH can be located in a parasellar region such as the cavernous sinus and might escape visualization during surgery. Only limited data have been published by other investigators who encountered similar cases. ${ }^{25,28}$ Of 11 patients in such cases in the two reports (cases in which the results of exploration at the time of initial hemihypophysectomy were negative), eight had remission of the hypercortisolemic state after subsequent total hypophysectomy. ${ }^{25,28}$ Thus, in light of the high morbidity and mortality rates associated with Cushing disease, we believe the approach is justified and reasonable as long as there are definitive data demonstrating a pituitary source of ACTH hypersecretion and hypopituitarism is an acceptable risk in the specific cases in question.

\section{Conclusions and Recommendations}

An algorithm for the perioperative management of patients with Cushing disease is presented in Fig. 5. Pituitary adenomectomy is the standard approach for initial management. The interaction and communication between all members of the management team are important in the final outcome of these patients. The success of this intervention depends to a large degree on the experience and expertise of the management team as well as the location, extent, and the invasive nature of the adenoma. Establishing an accurate diagnosis is the first step towards optimal management of patients with this disease. Routine administration of glucocorticoids in the perioperative period is not warranted nor is it clinically indicated. The routine use of perioperative glucocorticoids would confound the interpretation of clinical as well as biochemical data obtained after surgery. Frequent determinations of serum cortisol levels as well as close clinical monitoring for signs and symptoms of adrenal insufficiency are necessary. Glucocorticoids should be administered when serum cortisol levels fall below $2 \mu \mathrm{g} / \mathrm{dl}$ or when clinical signs of adrenal insufficiency are encountered. The absence of hypocortisolism postoperatively should prompt a reassesment of the diagnosis, evaluation of potential confounding elements, and consideration of repeated surgery.

\section{Acknowledgments}

We thank all our referring physicians and the nursing staff at the Case Medical Center neurointensive care unit and neurosurgical service for their help in obtaining the blood samples in a timely manner. 


\section{References}

1. Arafah BM: Reversible hypopituitarism in patients with large nonfunctioning pituitary adenomas. J Clin Endocrinol Metab 62: 1173-1179, 1986

2. Arafah BM, Kailani SH, Nekl KE, Gold RD, Selman WR: Immediate recovery of pituitary function after transsphenoidal resection of pituitary macroadenomas. J Clin Endocrinol Metab 79: 348-354, 1994

3. Arafah BM, Nasrallah MP: Pituitary tumors: pathophysiology, clinical manifestations and management. Endocr Relat Cancer 8:287-305, 2001

4. Arafah BM, Pearson OH (eds): Cushing's Disease. Philadelphia: WB Saunders Company, 1984

5. Banerjee A, Martin N, Dhillo W, Roncaroli F, Meeran K: Use of the dexamethasone-suppressed corticotrophin-releasing hormone test to predict cure in patients with Cushing's disease following pituitary surgery. Endocrine Abstracts 13: P243, 2007

6. Benveniste RJ, King WA, Walsh J, Lee JS, Delman BN, Post KD: Repeated transsphenoidal surgery to treat recurrent or residual pituitary adenoma. J Neurosurg 102:1004-1012, 2005

7. Chee GH, Mathias DB, James RA, Kendall-Taylor P: Transsphenoidal pituitary surgery in Cushing's disease: can we predict outcome? Clin Endocrinol (Oxf) 54:617-626, 2001

8. Chen JC, Amar AP, Choi S, Singer P, Couldwell WT, Weiss MH: Transsphenoidal microsurgical treatment of Cushing disease: postoperative assessment of surgical efficacy by application of an overnight low-dose dexamethasone suppression test. J Neurosurg 98:967-973, 2003

9. Coe CL, Murai JT, Wiener SG, Levine S, Siiteri PK: Rapid cortisol and corticosteroid-binding globulin responses during pregnancy and after estrogen administration in the squirrel monkey. Endocrinology 118:435-440, 1986

10. Esposito F, Dusick JR, Cohan P, Moftakhar P, McArthur D, Wang $\mathrm{C}$, et al: Clinical review: Early morning cortisol levels as a predictor of remission after transsphenoidal surgery for Cushing's disease. J Clin Endocrinol Metab 91:7-13, 2006

11. Estrada J, Garcia-Uria J, Lamas C, Alfaro J, Lucas T, Diez S, et al: The complete normalization of the adrenocortical function as the criterion of cure after transsphenoidal surgery for Cushing's disease. J Clin Endocrinol Metab 86:5695-5699, 2001

12. Findling JW, Raff H: Cushing's Syndrome: important issues in diagnosis and management. J Clin Endocrinol Metab 91:3746-3753, 2006

13. Friedman RB, Oldfield EH, Nieman LK, Chrousos GP, Doppman JL, Cutler GB Jr, et al: Repeat transsphenoidal surgery for Cushing's disease. J Neurosurg 71:520-527, 1989

14. Graham KE, Samuels MH, Raff H, Barnwell SL, Cook DM: Intraoperative adrenocorticotropin levels during transsphenoidal surgery for Cushing's disease do not predict cure. J Clin Endocrinol Metab 82:1776-1779, 1997

15. Hammer GD, Tyrrell JB, Lamborn KR, Applebury CB, Hannegan ET, Bell S, et al: Transsphenoidal microsurgery for Cushing's disease: initial outcome and long-term results. J Clin Endocrinol Metab 89:6348-6357, 2004

16. Hamrahian AH, El-Mallawany NK, Arafah BM: Evaluation and management of pituitary-adrenal function after pituitary surgery. The Endocrinologist 9:16-24, 1999

17. Hamrahian AH, Oseni TS, Arafah BM: Measurements of serum free cortisol in critically ill patients. $N$ Engl J Med 350: 1629-1638, 2004

18. Hout WM, Arafah BM, Salazar R, Selman W: Evaluation of the hypothalamic-pituitary-adrenal axis immediately after pituitary adenomectomy: is perioperative steroid therapy necessary? J Clin Endocrinol Metab 66:1208-1212, 1988

19. Höybye C, Grenbäck E, Thorén M, Hulting AL, Lundblad L, von Holst H, et al: Transsphenoidal surgery in Cushing disease: 10 years of experience in 34 consecutive cases. J Neurosurg 100:634-638, 2004
20. Imaki T, Tsushima T, Hizuka N, Odagiri E, Murata Y, Suda T, et al: Postoperative plasma cortisol levels predict long-term outcome in patients with Cushing's disease and determine which patients should be treated with pituitary irradiation after surgery. Endocr J 48:53-62, 2001

21. Inder WJ, Espiner EA, MacFarlane MR: Outcome from surgical management of secretory pituitary adenomas in Christchurch, New Zealand. Intern Med J 33:168-173, 2003

22. Inder WJ, Hunt PJ: Glucocorticoid replacement in pituitary surgery: guidelines for perioperative assessment and management. J Clin Endocrinol Metab 87:2745-2750, 2002

23. Klose M, Jorgensen K, Kristensen LO: Characteristics of recovery of adrenocortical function after treatment for Cushing's syndrome due to pituitary or adrenal adenomas. Clin Endocrinol (Oxf) 61:394-399, 2004

24. Lamberts SW, van der Lely AJ, de Herder WW: Transsphenoidal selective adenomectomy is the treatment of choice in patients with Cushing's disease. Considerations concerning preoperative medical treatment and the long-term follow-up. J Clin Endocrinol Metab 80:3111-3113, 1995

25. Locatelli M, Vance ML, Laws ER: Clinical review: the strategy of immediate reoperation for transsphenoidal surgery for Cushing's disease. J Clin Endocrinol Metab 90:5478-5482, 2005

26. McCance DR, Besser M, Atkinson AB: Assessment of cure after transsphenoidal surgery for Cushing's disease. Clin Endocrinol (Oxf) 44:1-6, 1996

27. Orbach O, Schussler GC: Increased serum cortisol binding in chronic active hepatitis. Am J Med 86:39-42, 1989

28. Ram Z, Nieman LK, Cutler GB Jr, Chrousos GP, Doppman JL, Oldfield EH: Early repeat surgery for persistent Cushing's disease. J Neurosurg 80:37-45, 1994

29. Rollin GA, Ferreira NP, Junges M, Gross JL, Czepielewski MA: Dynamics of serum cortisol levels after transsphenoidal surgery in a cohort of patients with Cushing's disease. J Clin Endocrinol Metab 89:1131-1139, 2004

30. Simmons NE, Alden TD, Thorner MO, Laws ER Jr: Serum cortisol response to transsphenoidal surgery for Cushing disease. J Neurosurg 95:1-8, 2001

31. Sonino N, Boscaro M, Fallo F: Pharmacologic management of Cushing syndrome: new targets for therapy. Treat Endocrinol 4: 87-94, 2005

32. Stalla GK, Stalla J, Huber M, Loeffler JP, Höllt V, von Werder K, et al: Ketoconazole inhibits corticotropic cell function in vitro. Endocrinology 122:618-623, 1988

33. Trainer PJ, Lawrie HS, Verhelst J, Howlett TA, Lowe DG, Grossman AB, et al: Transsphenoidal resection in Cushing's disease: undetectable serum cortisol as the definition of successful treatment. Clin Endocrinol (Oxf) 38:73-78, 1993

34. Vance ML: Perioperative management of patients undergoing pituitary surgery. Endocrinol Metab Clin North Am 32: 355-365, 2003

35. Yap LB, Turner HE, Adams CBT, Wass JAH: Undetectable postoperative cortisol does not always predict long-term remission in Cushing's disease: a single centre audit. Clin Endocrinol (Oxf) 56:25-31, 2002

36. Zayour DH, Selman WR, Arafah BM: Extreme elevation of intrasellar pressure in patients with pituitary tumor apoplexy: relation to pituitary function. J Clin Endocrinol Metab 89: 5649-5654, 2004

Manuscript submitted July 5, 2007.

Accepted August 13, 2007.

Address correspondence to: Baha M. Arafah, M.D., Division of Endocrinology, University Hospitals/Case Medical Center, 11100 Euclid Avenue, Cleveland, Ohio 44106. email: baha.arafah@ case.edu. 